\section{Supposed Direct Spectroscopic Observation of the "Oxygen-Transporting Ferment"}

THE supposition as to the hæmatin nature of oxidase (or Warburg oxygen transporting ferment) has been based so far only on the photochemical absorption spectrum obtained from the study of the effect of light, of definite energy and different wavelengths, on the oxygen uptake of cells poisoned with carbon monoxide. The concentration of the oxidase in cells is supposed to be too low for the direct spectroscopic observation of this enzyme even in the actively respiring yeast cells ${ }^{1}$.

It was announced recently, however, by Warburg and Negelein ${ }^{2}$ that in Bacterium pasteurianum, which has a very high respiratory activity (at $38^{\circ} \mathrm{C} . Q_{0_{3}}=$ $1,000)$, the absorption spectrum of the ferment can be seen by the direct spectroscopic examination of a thick suspension of these organisms illuminated with a strong light. Such examination, in the absence of oxygen, reveals, according to them, in addition to the two $\alpha$-bands of cytochrome (band $a$ being absent), a diffuse band in the yellow, which they believe to be the $\alpha$-band of the ferment in its reduced state. When the suspension is saturated with carbon monoxide a carbon monoxide-ferment compound is formed, the band becomes sharper and lies at $593 \mathrm{m \mu}$, that is, in the same place as the $\alpha$-band of the photochemjcal absorption spectrum obtained by Kubowitz and $\mathrm{Haas}^{3}$. When the suspension is saturated with oxygen, this band, as well as the cytochrome bands, disappears. If, however, the suspension is saturated with oxygen in the presence of cyanide, the bands of reduced eytochrome remain visible, while a new band at about $640 \mathrm{~m} \mu$ appears. This band, according to Warburg and Negelein, corresponds to a "methæmoglobin-like" compound of the ferment, with trivalent iron probably combined with cyanide.

The study of cytochrome in cells of different organisms has led me, however, to the conclusion that, although the observations of these authors are on the whole correct, their interpretations are certainly wrong. This conclusion is supported by several observations :-

(1) B. pasteurianum is a very polymorphic organism and the absorption spectrum of its cytochrome varies with the strain of the organism*, the culture medium and the age of the culture. Young cultures (grown on peptone-broth-sugar-alcohol agar) show a strong component $c$ and a very feeble $b$ with its $\alpha$-band more or less fused with that of $c$. The band $a$ which in baker's yeast lies at $603 \mathrm{~m} \mu$ is missing, being here replaced by two very faint shadings one at about $590 \mathrm{~m} \mu$ and the other, which can be seen in cultures of some strains only, lying at about $635 \mathrm{~m} \mu$. The intensities of these bands vary with the culture. All these bands disappear on shaking the suspension with air. The effect of carbon monoxide on the absorption spectrum also varies with the culture : in some suspensions saturated with carbon monoxide the shading at $595 \mathrm{~m} \mu$ becomes more distinct (as was found by Warburg and Negelein); in other cultures, in addition to this change, the component $b$ and a portion of $c$ also combine with carbon monoxide; and finally, in some other cultures, carbon monoxide has no visible effect on the absorp. tion spectrum. In other words, the property of the component with the band in the yellow to combine

* One of the strains of Bacterium pasteurianum was kindly given to me by Prof. A. J. Kluyver, of Delft. with carbon monoxide can be shared by other components of cytochrome.

(2) The shadings in the yellow at about $590 \mathrm{~m} \mu$ and in the red at about $630 \mathrm{~m} \mu$ or $636 \mathrm{~m} \mu$ (replacing the typical band $a$ of cytochrome) are found in other micro-organisms such as Bacillus proteus and Azotobacter chroococcum, where they can be easily seen on examination with a small dispersion prism spectroscope. Here the band in the red $(630 \mathrm{~m} \mu)$ is even more distinct than in $B$. pasteurianum. A similar band has been previously described by Yaoi and Tamiya ${ }^{4}$ in several strains of $B$. dysenterio and $B$. coli, which are also devoid of a typical band $a$ of cytochrome. Moreover, a diffuse band in the yellow at $588 \mathrm{~m} \mu$ or $592 \mathrm{~m} \mu$ exists also in brewer's yeast ${ }^{5}$ where it replaces the usual band $a$ of cytochrome, which in baker's yeast lies at $603 \mathrm{~m} \mu$.

(3) If the direct spectroscopic demonstration of the "respiratory ferment" depends on the respiratory activity of the organism, Azotobacter, which at $28^{\circ} \mathrm{C}$. has $Q_{\mathrm{O}_{2}}=8,000$, would naturally form the most suitable material for such demonstration ${ }^{6}$. The thick suspension of this organism does not reveal, however, any visible change even if the suspension is saturated with pure carbon monoxide. Neither the shading at $590 \mathrm{~m} \mu$ nor the other bands are affected by carbon monoxide.

(4) In B. proteus and in Azotobacter, where the band in the red $(630 \mathrm{~m} \mu)$ can be clearly seen, like all other bands of cytochrome, it fades away on shaking the suspension with air (or is replaced by a very faint shading at $645 \mathrm{~m} \mu$ ), and it reappears on standing or on reduction with sodium hydrosulphite. The band in the red belongs, therefore, to a reduced or ferrous state of the pigment and not to an oxidised com. pound with a trivalent iron atom.

(5) On acidifying gradually with normal hydrochloric acid, a thick (50 per cent) suspension of baker's yeast containing a little caprylic alcohol, and shaking the suspension with air, a stage is reached when, on reduction with sodium hydrosulphite, the band $a$ (lying usually at $603 \mathrm{mu}$ ) shifts towards the short wave end of the spectrum and becomes replaced by a shading in the yellow. This shading becomes distinctly darker and sharper only in the presence of carbon monoxide, forming a band at about $596 \mathrm{~m} \mu$, that is, in the region of the $\alpha$-band of the photochemical absorption spectrum obtained by Kubowitz and Haas. In other words, a compound with the absorption band and other properties similar to those of the compound found in $B$. pas teurianum can be obtained from the component $a$ of cytochrome, which as we know is extremely fragile and easily changes its absorption spectrum and other properties.

These experiments show clearly that the shading in the yellow $(590 \mathrm{~m} \mu)$ and the band in the red $(630 \mathrm{~m} \mu$ or $636 \mathrm{~m} \mu$ ) are not the absorption bands of the oxygen transporting enzyme but the bands of some derivatives of cytochrome and probably of its component $a$.

Molteno Institute,

D. KEILIN.

University of Cambridge. Oct. 17.

1 Warburg, O., and Kubowitz, F., Bioch. Z., 203, 95; 1928.

Warburg., O., and Negelein, E., ibid., 262, 237; 1933.

- Kubowitz, F., and Haas, E., ibid., 255, 247 ; 1932.

- Xaoi, H., and Tamiya, H., Proc. Imp. Acad. Japan, 4, 436 ; 1928.

'Fink, H., Z. physiol. Chem., 210, 197 ; 1932.

-Meyeriof, O., and Schulz, W., Biosh. Z., 250, 35 ; 1932. 